BULLETIN Bulletin hispanique

HISPANIQUE Université Michel de Montaigne Bordeaux

$119-2 \mid 2017$

La Égloga renacentista en el Reino de Nápoles

\title{
Cedros de nuestro Líbano
}

sacralidad y sentido de pertenencia en un poema de Rosalía de Castro

María do Cebreiro Rábade Villar

\section{(2) OpenEdition}

Journals

Edición electrónica

URL: http://journals.openedition.org/bulletinhispanique/5195

DOI: 10.4000/bulletinhispanique.5195

ISSN: 1775-3821

Editor

Presses universitaires de Bordeaux

Edición impresa

Fecha de publicación: 1 diciembre 2017

Paginación: 715-732

ISBN: 979-10-300-0218-8

ISSN: 0007-4640

Referencia electrónica

María do Cebreiro Rábade Villar, «Cedros de nuestro Libano», Bulletin hispanique [En línea], 119-2 | 2017,

Publicado el 28 diciembre 2020, consultado el 13 enero 2021. URL: http://journals.openedition.org/

bulletinhispanique/5195 ; DOI: https://doi.org/10.4000/bulletinhispanique.5195 


\title{
Cedros de nuestro Líbano: sacralidad y sentido de pertenencia en un poema de Rosalía de Castro ${ }^{1}$
}

\author{
María do Cebreiro Rábade Villar \\ Universidad de Santiago de Compostela
}

L'objectif de cet article est d'explorer la signification des arbres dans l'œuvre de Rosalía de Castro à partir d'un examen minutieux de l'image " cèdres de notre Liban " du poème " iJamás lo olvidaré...!" (En las orillas del Sar, 1884). En analysant jusqu’à quel point les arbres coupés fonctionnent comme mémoire d'une perte, il est permis de reconnaître dans le poème l'empreinte de différents discours culturels.

Mots-clés: Rosalía de Castro, culture et territoire, arbres, nationalisme et religion, orientalisme péninsulaire.

El objetivo del artículo es explorar el significado de los árboles en la literatura rosaliana, a partir de un atento examen de la imagen "cedros de nuestro Líbano» del poema «jJamás lo olvidaré...!» (En las orillas del Sar, 1884). Al analizar hasta qué punto los árboles cortados funcionan como memoriales de una pérdida, se hace posible reconocer en el poema la impronta de distintos discursos culturales.

Palabras-clave: Rosalía de Castro, cultura y territorio, árboles, nacionalismo y religión, orientalismo peninsular.

The article intends to explore the meaning of trees in Rosalíade Cstro's literature, from a careful examination of the image "cedars of our Lebanon" in the poem "iJamás lo olvidaré...!" ("En las orillas del Sar', 1884). When analysing to what extent cut-off trees act as memorials of a loss, it is possible to recognise in the poem the mark of different cultural discourses.

Keywords: Rosalía de Castro, culture and territory, trees, nationalism and religion, peninsular orientalism.

1. Deseo agradecer al investigador Román García Alberte su ayuda en el desarrollo de este trabajo, tanto en su concepción teórica como en su ejecución escrita. Especialmente útil resultó su colaboración en el acopio de fuentes documentales, sobre todo de aquellas relacionadas con la teoría del nacionalismo y con la historia del Líbano. 
D entro del ámbito de la teoría del nacionalismo el valor concedido al imaginario territorial como símbolo de la identidad colectiva constituye una zona de fuerte consenso crítico. Reformulando parcialmente las hipótesis de Liisa Malkki, Umut Özkirimli se ha referido a este fenómeno como «nacionalización territorial del espacio» y ha puesto de relieve el papel de los escritores e intelectuales en este proceso. Como refrenda una larga tradición cultural (al menos desde el idilio pastoral clásico hasta el romanticismo), el paisaje, en tanto construcción cultural de la naturaleza, ha sido uno de los elementos priorizados en la estetización del territorio. Dentro de éste las metáforas botánicas, específicamente las arbóreas, han jugado una función muy relevante. ${ }^{2}$ En palabras de Özkirimli: "Not surprisingly, nations and national identities too are conceptualized in terms of roots, trees, origins, ancestries, racial lines, evolutions, developments, or other familiar, essentializing images. What they all share ... is a genealogical form or thought, which is peculiarly arborescent.» (181). En la constitución de las modernas naciones, el fenómeno que Özkirimli conceptualiza como "a genealogical form or thought, which is peculiarly arborescent» resulta indisociable de la emergencia de la ciencia biológica, tal y como fue concebida por Michel Foucault a partir del libro Las palabras y las cosas. En un proceso que tiene su inicio en la segunda mitad del siglo XVIII y que culminará en la primera mitad del siglo XIX, el pensamiento occidental asistió a una profunda transformación epistémica, que dio lugar a la clausura de la historia natural y a la emergencia de la biología. La imagen del árbol, en tanto que metáfora científica, constituye una cifra decisiva de este proceso.

Según Foucault, el cambio de paradigma tuvo que ver precisamente con la reorganización categorial (taxonómica en Cuvier y explícitamente genealógica en Darwin) de un saber sobre la naturaleza. Esta transformación se relaciona de un modo muy relevante con la emergencia de la categoría de población y el modo en que ésta afecta la conceptualización de las especies animales o arbóreas. Otro cambio no menos decisivo fue que en el nuevo paradigma las funciones de los organismos vivos se hacen depender de las condiciones de existencia: nacimiento, desarrollo y mortalidad. Aquello que, según Foucault introduce la biología en el ámbito del saber es la caracterización del ser vivo como aquel que es «capaz de morir» (Pavesi 93).

La mortalidad de los árboles, su constitución como población viva y su significado para la comunidad, constituyen los ejes poético y político de dos poemas de Rosalía de Castro, situados en el libro En las orillas del Sar

2. Para explicar el rendimiento de la metáfora del arraigo en el ideario nacionalista, Malkki remite a la naturalización específicamente arbórea de la relación entre las poblaciones humanas y el lugar que habitan: «the naturalizing of the links between people and place is routinely conceived in specifically botanical metaphors. That is, people are often thought of, and think of themselves, as being rooted in place and as deriving their identity from that rootedness... They are specifically arborescent in form.» (Liisa Malkki, «National Geographic: The Rooting of Peoples and the Territorialization», Becoming National. A Reader, New York \& Oxford, Oxford University Press, 1996, pp. 434-53, p. 342). 
(1884). «jamás lo olvidaré...!» y «Los robles» tienen su origen enunciativo en la deforestación del bosque que circundaba el monasterio de Conxo, situado en la periferia de la ciudad de Santiago de Compostela (Rodríguez 408). En los dos textos el propósito de la voz poética es dar testimonio de los efectos de la tala en los bosques autóctonos, representados sobre todo en la figura poblacional de los robledales, de los castańos y de los pinos. Pero es en la comparación entre el cedro y los árboles gallegos, que tiene lugar en el poema ¡Jamás lo olvidaré...!, donde la autora encontrará un poderoso mecanismo de legitimación cultural, basado, como veremos más adelante, en el valor simbólico y religioso concedido por su círculo de sociabilidad a los referentes orientales. Por otra parte, al poner de relieve el modo en que funciona la economía política de la deforestación, Rosalía de Castro reconoce en estos poemas la función simbólica (artística, religiosa, mítica y cultural) de los árboles en la constitución histórica de la comunidad.

Resulta muy revelador consignar la evolución del motivo de los cedros en el corpus rosaliano, examinando las diferencias que afectan a su modo de representación, lógicamente muy dependientes del contexto semántico y axiológico al que se adscriben. Dentro de su producción, el sintagma «cedros del Líbano» aparece por primera vez en el capítulo octavo de su segunda novela (Flavio, 1861). El joven Flavio, que había escapado de la casa paterna tomando por divisa el lema «la verdadera patria del hombre es el mundo entero» (Castro, Flavio 219), y se dispone a emprender un tortuoso peregrinaje vital, irrumpe en un baile campesino celebrado en el claro de un bosque. Es en la descripción de los movimientos masculinos donde la narradora sitúa la expresión «cedros del Líbano», atribuida a la tradición bíblica del Cantar de los Cantares, cuya enunciación femenina enfatiza:

Sus ligeros pies tocaban apenas el suelo, resbalaban a compás igual sobre una tersa superficie, uno en pos de otros, como si se atrajesen unos a otros por medio de un oculto y misterioso poder. Las cabezas, graciosamente modeladas, de aquellas hermosas mujeres se inclinaban, mientras las de los hombres se levantaban como cedros del Líbano, según la poética expresión de la Esposa de los Cantares. Comprendíase fácilmente que aquellos seres, presa del abandono y de la embriaguez de la danza, debían sentir que sus corazones palpitaban unidos, y que, como dos lágrimas que resbalasen juntas, debían confundirse, aliento con aliento, la dulce mirada de la mujer con la mirada atrevida y embriagadora del hombre. (Castro, Flavio 241)

El baile colectivo descrito en este pasaje de Flavio opera como una suerte de iniciación, capaz de determinar la conflictiva entrada del protagonista en el mundo social. En su camino hacia la vida adulta, el muchacho empieza por observar que el principio de división sexual puede ser simbólicamente reemplazado por el de la fusión de los cuerpos («aquellos seres presa del abandono y de la embriaguez de la danza.») Al situar este rito de paso en una escena de baile, la narradora acentúa de modo simultáneo la existencia de la ley social y su disolución momentánea en el abrazo de las parejas. Cabría observar, además, que el baile no es una danza popular, sino un vals. Sin embargo, la 
escena no tiene lugar en un espacio de sociabilidad urbana, ni un escenario ligado a la esfera del mundo cortesano (por ejemplo, un salón), sino en el corazón del mundo natural. Por medio de esta elección, el texto refuerza la peculiar y compleja posición de la literatura rosaliana en el conflicto entre la civilización y la naturaleza (García Candeira 2013).

Los cedros aparecerán de nuevo en el libro tercero de Follas novas («Varia»), publicado en 1881 pero cuya fecha de escritura, según autores como Catherine Davies (343), habría que retrotraer al decenio anterior. De nuevo, el árbol hará su aparición en un contexto significativo. Un personaje solitario (en este caso, una anciana a quien la comunidad toma por bruja) vive en el medio de un bosque descrito en el poema a partir de su configuración arbórea: olmos, bojes, laureles, limoneros y naranjos. En el poema los cedros no son presentados como un árbol entre los demás, ni como una metáfora antropomórfica, sino en su pura materialidad funcional. En concreto, su función es operar como testimonio de la solidez e inviolabilidad de un determinado espacio, que, pese a su humildad y a su presencia amenazadora, es caracterizado como si fuese un templo:

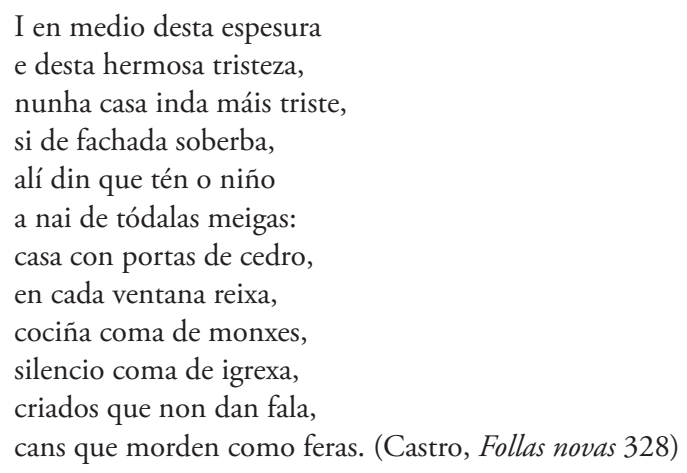

En una estrofa muy característica de la tensión ideológica y estética que atraviesa a menudo la obra rosaliana, la autora oscila entre dos ejes de valoración. En consonancia con los poderes maléficos que la comunidad atribuye a la mujer, la hablante afirma que sus perros guardianes «morden como feras». Por otra parte, no renuncia a encarecer de modo muy expresivo la nobleza de la casa («fachada soberba») y la sacralidad del lugar («silencio coma de igrexa»). En auxilio de este segundo eje interpretativo opera la venerable tradición según la cual el templo de Salomón había sido construido con madera de cedro ${ }^{3}$. En

3. El carácter fuerte y noble de la madera del cedro ha dado lugar a numerosos testimonios historiográficos y literarios, desde el muy remoto de Diodoro Sículo (apud Nadia Zaafer Chaban, La imagen del Líbano en los escritos de viaje españoles de la segunda mitad del siglo XIX, Madrid, Universidad Autónoma, 1988, p. 75) hasta la obra de autores libaneses contemporáneos como Khalil Gibran (Elise Salem, Constructing Libanon. A Century of Literary Narratives, Gainesville, University Press of Florida, 2003, p. 20, p. 22). En su estudio sobre la deforestación del Monte Líbano, Marvin W. Mikesell parte asimismo de una mención del Gilgamesh, sin duda uno de los testimonios más antiguos de este motivo: «who is this that has violated my woods and cut down my cedar?» (Marvin W. Mikesell «The Deforestation of Mount Lebanon», The Geographical Review, 
tanto garantía de la sacralidad del lugar, el cedro anticipa de hecho el sentido de los últimos versos del poema, desmintiendo con rotundidad la impresión de que la casa descrita es un locus horridus. Al sostener que el mundo suele absolver a los culpables y condenar a los inocentes, la voz toma posición en favor del personaje y condena implícitamente a la comunidad, a quien hace responsable de su exclusión ${ }^{4}$. Por segunda vez, la imagen del cedro permite poner en relación las reglas del mundo social (para Rosalía de Castro, a menudo crueles y falibles) con los principios del mundo natural, mediatizados culturalmente por referencias de fuerta impronta mítica y simbólica.

Pero será en el poema «jJamás lo olvidaré...!», publicado por primera vez en 1882 en el periódico La Nación Española (Rodríguez 408) y reeditado en el volumen En las orillas del Sar (1884), cuando los cedros adquieran un valor político explícito, en íntima conexión con la defensa de un medio natural amenazado. Desde el punto de vista retórico, el texto se asienta en el postulado de dos principios relacionados entre sí: a) el vínculo entre identidad y recuerdo (que es posible relacionar con el complejo mito-símbolo-memoria, tal y como ha sido caracterizado por Anthony Smith), y b) el vínculo entre la sacralidad y el sentido de pertenencia. Desde el punto de vista de la configuración de un imaginario colectivo, la alianza entre estos dos elementos tiene su origen en un empleo estratégico de la teoría platónica de la anamnesis, que sitúa en la memoria el origen del verdadero conocimiento, y cuyas reverberaciones en la poética del siglo XIX llegaron a autores tan estimados por Rosalía de Castro como Edgar Allan Poe.

La conexión entre la creación poética y el acto de rememorar resuena desde el título del poema. Tanto desde el punto de vista conceptual como tonal, «jJamás lo olvidaré...!» prefigura un sujeto intensamente afectado por dos cualidades: la testimonial y la expresiva. La capacidad testimonial del sujeto encuentra su fundamento en la dimensión narrativa del poema, llamado a actuar, en el plano simbólico, como prueba judicial de una denuncia. El recuerdo de un yotestigo, capaz de dar fe de la existencia de un bosque y de su significado para la comunidad, se articula como la fuente de sensibilidad y de conocimiento más legítima, y por lo tanto también como un mecanismo apto para condenar la falta cometida. En segundo lugar, «jJamás lo olvidaré...!» asienta su fuerza retórica en la capacidad expresiva del sujeto, acentuada en virtud de diferentes motivos de apariencia sentimental que esconden a su vez complejas valencias

40.1, 1969, p. 1-28, p. 1). Entre otros referentes bíblicos, sería posible aducir a Jeremías (22, 23), Isaías (10, 34), Ezequiel (12), Zacarias (21, 1), los Salmos (92, 13), el Eclesiastés (24, 13), el Libro de los Reyes $(\mathrm{V}, 8,9)$ y Esdras $(3,7)$. A lo largo de la historia, los cristianos maronitas han otorgado al cedro un claro valor político y etnorreligioso, considerándolo, como ha anotado irónicamente As'ad Abukhalil, "a special gift to a special people in a special land.» (As'ad Abukhalil, Historical Dictionary of Lebanon, Lanham, The Scarecrow Press, 1998. s. v. «Cedar of Lebanon»).

4. "Ali a viron negra e fraca / coma unha gata famenta, / no máis san e máis frorido / da hermosa terra gallega. I I estes mals que nos afrixen / din que todos veñen dela... / Mais socede nesta vida / que os que tén culpa na levan!» (Rosalía de Castro, Follas novas, en Marina Mayoral, Obras completas II, Madrid, Turner, 1993, p. 321). 
ideológicas. Lo peculiar en la aplicación rosaliana de la anamnesis es el hecho de que la subjetividad romántica es explícitamente sujetada al sentido histórico, de modo que la memoria personal acaba por confluir con la memoria cultural y la hablante asume (como veremos, no sin reticencias) el papel de portavoz de la comunidad.

Para ilustrarlo, podemos considerar la sutil transición que el poema opera en una de sus estrofas, por medio de la cual un escenario salvaje es convertido en un jardín. En una brillante demostración de la diferencia filosófica entre lo sublime y lo bello en la naturaleza, el bosque autóctono de las primeras estrofas («esas selvas agrestes, esos bosques / seculares y hermosos» (Castro, En las orillas... 482) dará paso de pronto a un edén de resonancias bíblicas. $\mathrm{Y}$ el elemento que opera como punto de inflexión entre ambos espacios es, nuevamente, el cedro. Así, una vez ha descrito el efecto de la tala en los árboles milenarios (entre ellos, los castańos a los que denomina «cedros de nuestro Líbano» (Castro, En las orillas... 483) la voz se refiere a todos aquellos elementos del mundo natural que no regresarán al escenario destruido por la tala. $Y$ en este paisaje negativo, que tiene únicamente lugar en la memoria personal de quien describe la escena, la hablante enumera:

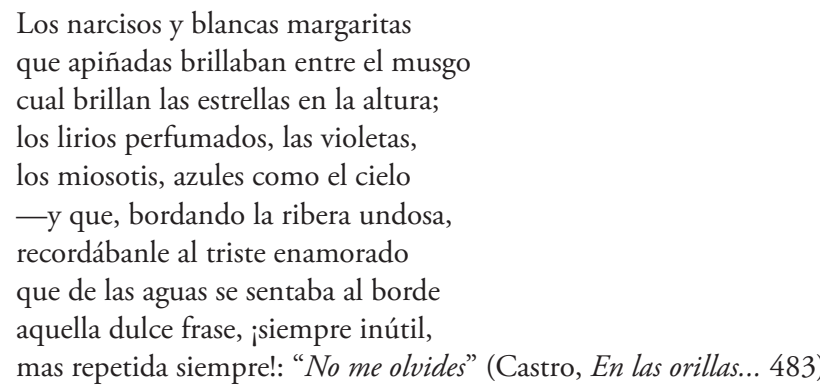

Se trata de una enumeración extraña en quien afirmó ser ajena a cierta poesía femenina de su tiempo, aquella que identificaba el alma de las mujeres con su capacidad para celebrar la belleza de «las palomas y las flores», tal y como se afirma en el poema prologal de Follas novas. Pero esta enumeración no es un telón de fondo. Antes bien, nos sitúa ante uno de los peculiares ejemplos de traducción cultural y de descontextualización semántica que ofrece la obra de Rosalía de Castro. Si en la novela Flavio, rindiendo homenaje a Edgar Allan Poe, la autora atribuía a la pequeña ciudad de Santiago de Compostela la fisonomía de una gran metrópolis (Rábade, «Spleen...»), «jamás lo olvidaré...!» convierte un bosque autóctono en un jardín digno de figurar en el Cantar de los Cantares. Entre todas las flores mencionadas en el pasaje (narcisos, margaritas, lirios y miosotis), merecen especial atención los «lirios perfumados, pues junto a la rosa del desierto, el lirio del valle es la imagen elegida por la Esposa del Cantar para autocaracterizarse («Yo soy un narciso del Sarón, / un lirio de la vega», Alonso ed. 15), y también la imagen elegida por el amante para saludarla: "Comparar 
el lirio a las zarzas / es comparar mi amiga a las muchachas.» (Alonso ed. 15) ${ }^{5}$. En glosa a estos versículos, Fray Luis de León -el autor por quien, seguramente en la edición de Fray Antolín Merino (1806), Rosalía de Castro accedería a la traducción del libro bíblico- anota:

Esto dice la Esposa del Esposo, como si más claro dijese: «Yo soy rosa del campo, y tú, Esposo mío, lilio del valle.» En lo cual muestra cuán bien diga la hermosura del uno con la belleza del otro, y que, como se dice de los desposados, son para en uno; como lo son la rosa y el lilio, que juntos crece la gentileza de entrambos y agradan a la vista y dan olor más que cada uno por sí. Demás que siendo entrambas rústicas flores, cuadra bien la una con la otra, que la una es rosa del campo y la otra lilio de los valles, donde la naturaleza es la hortelana, que por estar el lugar más húmedo, está más fresco y de mejor parecer. (León 55)

La misma trama semántica, capaz de convertir el mundo natural en correlato del mundo emotivo, permite contextualizar los seis versos que la autora dedica a la flor del miosotis, denominada popularmente «nomeolvides». En el tratamiento de esta flor el texto difiere del valor positivo que le había conferido la poesía victoriana inglesa, tal y como había sido cristalizada en el conocido dístico de Tennyson: «I move the sweet forget-me-nots / That grow for happy lovers.» (124) Tal vez por interferencia con el mito de Narciso, en el poema de Rosalía de Castro el miosotis es contemplado por el enamorado en «la ribera undosa», referencia y emplazamiento que intensifican la negatividad especular y el solipsismo de la escena. Fiel a su acostumbrado pesimismo en la concepción del amor, a través de la voz del poema la autora parece sugerir que incluso los más puros sentimientos se deterioran y que en la vida humana la resistencia al paso del tiempo es inane, pues todo lo humano está condenado a desaparecer («aquella dulce frase, ¡siempre inútil, / mas repetida siempre!: "No me olvides”." (Castro, En las orillas... 483). De modo elegíaco, el nombre de la flor opera como memorial de un esplendor interrumpido en la tierra y como garantía de que, ante la ausencia, recordar equivale a existir. Esta figuración del mundo natural parece, en fin, ajena a todo realismo. En ella actúa más bien un deseo de enaltecimiento del objeto del canto, fundamentado en fuentes literarias y religiosas. Al igual que la mención de los lirios perfumados entroncaba con la unión espiritual entre la Esposa y el Esposo del Cantar bíblico, la leyenda del miosotis, vinculada a la materialidad del mundo físico, confiere a una anécdota de apariencia sentimental un valor espiritual profundo. Por medio de ella el texto intenta reforzar el valor concedido a la memoria como contrapeso (fallido pero de algún modo persistente) a la crueldad de las leyes del mundo.

5. En su tesis doctoral sobre la recepción de San Juan de la Cruz en los siglos XVI, XVII y XVIII, Mialdea Baena considera a Castro como el precedente más importante del entronque de la poesía mística con la modernidad lírica (José Antonio Mialdea Baena, La recepción de la obra literaria de San Juan de la Cruz en España durante los siglos XVII, XVIII y XIX, Córdoba, Universidad de Córdoba, 2002, p. 167-169). Como fundamento de su hipótesis, el autor examina la presencia de autores como San Juan de la Cruz y Fray Luis de León en los manuales de literatura de la segunda mitad del siglo XIX escritos por autores como Prudencio Mudarra y Párraga, Dalmiro Fernández y Oliva, Francisco Holgado y Toledo y José Coll y Vehí. 
Si incluso una pequeña flor silvestre puede ser el recordatorio de una pérdida, los troncos mutilados de los árboles actuarán como los verdaderos memoriales del texto. Caracterizados como soldados heridos y como botín de guerra en manos del enemigo, ellos son los elementos que, con mayor claridad, permiten reconocer el impacto de la catástrofe ecológica y su reconocimiento como profanación. Las flores, adscritas al territorio filosófico de lo bello, pueden vincularse al recuerdo (en un sentido personal) y ser usadas como ornato y elemento de sublimación del encuentro entre los enamorados. Situados al amparo de la categoría de lo sublime, los árboles, en cambio, trascienden el tiempo de la vida humana, señalando la posibilidad de entender la noción de memoria en un sentido transpersonal y, en cierto sentido, póstumo: «el árbol fuerte / de larga historia y de nudosas ramas / que es orgullo del suelo que le cría / con savia vigorosa, y monumento / que en sólo un día no levanta el hombre.» (Castro, En las orillas... 482)

Esa es la razón por la cual, tanto en este poema como en "Los robles», la voz emprenderá una monumentalización consciente del imaginario arbóreo, convirtiendo los bosques gallegos en aquello que Pierre Nora denominaría lugares de memoria. En consonancia con la sublimidad y con el carácter monumental atribuido a la naturaleza, en ambos textos adquirirá un papel muy relevante el uso de un lenguaje sagrado. Los «santos recuerdos» y la "sacra encina de los celtas» que evidencian la importancia de una dimensión sagrada en «Los robles» (Castro, En las orillas... 475-477) dan paso en "jamás lo olvidaré...!» a un imaginario de signo todavía más ardiente. Desde la primera estrofa, la catástrofe de la tala es remitida a la caída de los muros de Jerusalén (482). Al vincular la deforestación del bosque con la destrucción de las murallas de una ciudad, la autora vuelve a estrechar el vínculo entre naturaleza y civilización, dejando claro que una agresión al medio natural es también un acto de barbarie. Por otra parte, la conexión de la estrofa con el asedio a la ciudad sagrada entronca el texto con precedentes tan notables como la Gerusalemme liberata de Torquato Tasso, sobre la Cruzada del papa Urbano II. El eco remoto de la octava real en la primera estrofa (ocho versos endecasílabos, aunque en su estructura tímbrica no se sujeten al metro clásico) parece reforzar esta filiación literaria.

Tomada como origen legendario del éxodo, la referencia a Jerusalén permite tender relaciones con las tres antiguas culturas del libro, abriendo camino al ideario de la "Tierra prometida», de vasta influencia en la tradición judeocristiana. El valor simbólico de la ciudad para los cristianos cobrará nuevos matices en el siglo XIX. En los medios intelectuales españoles, la expansión de un cierto pensamiento de Cruzada correrá parejo al interés por revisitar el pasado musulmán de España, como lo testimonia tempranamente la Historia de la dominación de los árabes en España de José Antonio Conde (1820). Por su parte, el escritor compostelano Neira de Mosquera, fundador de la Academia de Santiago, y que en varios sentidos podría considerarse el mentor literario de 
la generación de Rosalía de Castro (Cabo Aseguinolaza y Rábade Villar 2013), transitó el tema en sus artículos periodísticos.

En la pieza titulada «Espańa árabe» (1842), Neira se refiere a la conquista de los territorios árabes desde la perspectiva del esplendor andalusí, llegando a contraponer la ventaja militar de los cristianos a la superioridad artística y científica de los musulmanes. A pesar de su posicionamiento inequívocamente cristiano, el tono del artículo remite al célebre dístico "Graecia capta ferum victorem cepit / et artes intulit agresti Latio» (Epistula ad Pisones II, 1, 156), en el que Horacio reconoce que, en su brillantez cultural, en el fondo Grecia fue quien conquistó a Roma. Considérese, en este sentido, el siguiente pasaje: «Perdidos y abandonados y reducidos a una dominación ahogada por las conquistas de los Reyes castellanos, solo tenían el recuerdo de sus victorias, de sus triunfos, de su poder, de su civilización, porque ellos han sido los que cultivaron con más afán las ciencias. La medicina tuvo un Averroes, y la química fue mimada por los laboriosos hijos de la Atenas de aquellos tiempos» (Neira 1842a 19). Incidentalmente, en este artículo el autor también hará referencia al célebre cedro de los mártires, árbol de origen libanés situado en el campo de Ahabul -rebautizado como Campo de los Mártires por los cristianos- y bajo el cual se creía que San Juan de la Cruz se había entregado a la meditación y a la escritura.

Pero es sin duda la pieza "Jerusalén. El Santo Sepulcro», del mismo año, el texto de Neira que permite dibujar con mayor claridad el contexto de pensamiento que precede a Jerusalén nombrado en "iJamás lo olvidaré...!». El artículo constituye una topografía detallada de los Lugares Santos, con referencias literarias muy pertinentes para establecer la genealogía poética del texto rosaliano. Su autor cita, por ejemplo, las palabras de Lamartine a propósito del santo sepulcro (Neira, «Jerusalén» 82) y se refiere al poeta como el «Lord Byron francés». La obra del propio Byron es, de hecho, una de las fuentes literarias más oportunas para la cabal intelección del texto rosaliano, dada la simpatía que Rosalía de Castro sintió siempre por el autor inglés y que declara abiertamente, entre otros pasajes, en su primera novela La hija del mar. En efecto, cuando Neira de Mosquera se refiere al Jordán, lo caracteriza como aquel río «a cuyas orillas Lord Byron -el poeta que compara Dumas a una borrasca, a una fantasmagoría, a un vaso de alabastro con una luz dentro- ha clamado por la religión del Ungido» (Neira, «Jerusalén» 82). Neira de Mosquera está haciendo referencia al poema «On Jordan's Banks» (nótese la semejanza con el título En las orillas del Sar, pero también con el anterior $A$ orillas del Ulla, de Alfredo Vicenti) donde Byron hace profesión de la fe cristiana como si se tratase de una revelación recibida en tierra enemiga:

On Jordan's banks the Arab's camels stray,

On Sion's hill the False One's votaries pray,

The Baal-adorer bows on Sinai's steep-

Yet there- even there-Oh God! thy thunders sleep: 


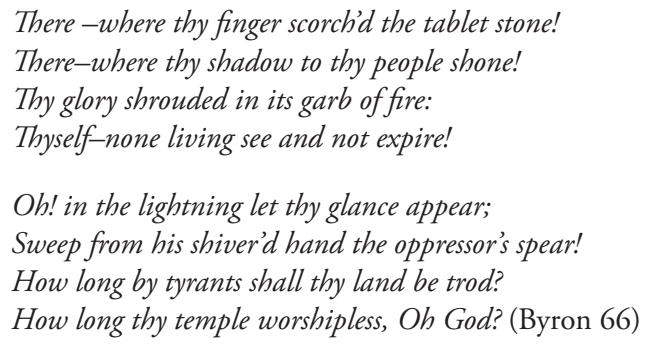

Neira de Mosquera se detiene en los peregrinos literarios que viajan a Jerusalén, pero los años ochenta del siglo XIX significarán el punto de arranque de un nuevo movimiento cultural: las peregrinaciones a Santiago de Compostela. El mismo año en que se publicó En las orillas del Sar, el Papa León XIII declaraba, por medio de la Bula «Deus Omnipotens», la autenticidad de las reliquias del Apóstol Santiago. El hallazgo de los restos de Santiago Zebedeo en la catedral compostelana -bajo el pontificado del cardenal Payá y Rico, responsable de la tala que denuncia el poema- permitió la equiparación simbólica entre Santiago, Roma y Jerusalén. Este hecho contribuye a explicar, asimismo, que algunos de los más significados viajeros por Oriente en la segunda mitad del siglo XIX sean de origen gallego o tengan relaciones con los medios intelectuales gallegos.

Resulta revelador de lo dicho el itinerario titulado Santiago, Jerusalén, Roma, diario de una peregrinación (1875), de José María Fernández Sánchez y Francisco Freire Barreiro, catedráticos de la Universidad de Santiago de Compostela, que incluye textos de Rosalía de Castro para ilustrar algunas descripciones de la catedral (Cabo Aseguinolaza, «Plotting out...»). Y no es este el único caso en el que el nombre de la autora gallega aparezca incidentalmente vinculado al de los viajeros por Oriente. Narciso Pérez Reoyo, médico residente en Galicia y miembro fundador de la Sociedad del Folklore Gallego (Barreiro 633), publica en el mismo año un Viaje a Egipto, Palestina y otros países de Oriente, y lo hacía en la imprenta luguesa Soto y Freire, donde Rosalía de Castro había publicado El caballero de las botas azules (1867). Se da la circunstancia de que a mediados de los ańos sesenta la autora había ejercido como preceptora literaria de su hija Narcisa Pérez Reoyo, amistad documentada en una carta exhumada por Carballo Calero ${ }^{6}$. También Manuel Murguía, intelectual

6. Algunos fragmentos de la carta, fechada el 4 de enero de 1866, muestran el ascendente que Rosalía de Castro ejercía sobre Narcisa Pérez Reoyo, uno de cuyos prólogos (escrito para el libro Cantos de la infancia) lleva la firma de Manuel Murguía. Véanse como ejemplo los siguientes pasajes: «Doy a U. las más expresivas gracias por la indulgencia con que ha mirado mis ensayos poéticos que de ningún modo merecen los elogios que U. hace de ellos, sin embargo me alegro en el alma de que sean del gusto de una persona tan autorizada como U. y que yo tanto aprecio [...] Quiera Dios que ellos me conduzcan algún día a escribir algo que se parezca a los numerosos escritos con que U. honra a nuestro país y a nuestro sexo. [...] Mucho agradezco también el ilustrado consejo de U. y procuraré seguirle en cuanto me lo permitan mis fuerzas, bien débiles por cierto para tan ardua empresa.» (apud Aurora López y Andrés Pociña, Rosalía de 
histórico del galleguismo y marido de Rosalía de Castro, había solicitado a Pérez Reoyo, por aquel entonces diputado, financiación para su magna Historia de Galicia (Barreiro 303). Por último, resulta interesante constatar que Adolfo de Rivadeneyra, autor de un Viaje de Ceylan a Damasco, Golfo Pérsico, Mesopotamia, Ruinas de Babilonia, Ninive y Palmira y Cartas sobre la Siria y la Isla de Ceylán (1871), actuase como impresor de la segunda edición madrileña de Cantares gallegos (1872).

Ahora bien, sería un error inferir que la obra de Rosalía de Castro se sitúa en una relación de determinismo o de causalidad con el contexto cultural e histórico que la rodeaba. La capacidad para superponer en su obra diferentes discursos estéticos y políticos, y para dialogar con ellos de un modo al tiempo comprometido y distanciado, es una de las características más notables de su producción. Podemos comprenderlo mejor si examinamos su peculiar tratamiento de los referentes religiosos en «jJamás lo olvidaré...!», y el modo en que funcionan como una tensa alegoría de la guerra ideológica que estaba librándose en el exterior del texto. Como ya hemos apuntado, la tala ordenada por el cardenal Payá y Rico tiene lugar en un robledal sito en el lugar de Conxo. La autora dedicó a este paraje, intensamente vinculado a su memoria emocional y a la historia gallega (en él tuvieron lugar acontecimientos tan relevantes para su generación como el denominado Banquete de Conxo o la fundación del primer hospital psiquiátrico de Galicia), varias páginas de su producción escrita, entre ellas su última novela El primer loco (Cabo Aseguinolaza, «Plotting out...).

En «jamás lo olvidaré...!», la voz refiere con detalle el trauma que en este bosque centenario originó la deforestación, y lo hace sin apelar únicamente a criterios paisajísticos o ecológicos, sino sugiriendo que destruir el medio natural es también destruir una forma de vida (Davies, "O mundo natural...»). En consonancia con su tendencia a favorecer la encarnación de los asuntos graves en las cosas pequeñas, Castro se detiene en las campanas, en el claustro y en la fuente y a «la aldeana hermosa / que lavaba sus lienzos en el agua / siempre brillante del pilón de piedra / que el roce de sus manos ha gastado / y hoy buscan de otra fuente la frescura.» (Castro, En las orillas... 484) Pero el idilio pastoral se verá interrumpido en la siguiente estrofa. Muy clara resulta la referencia al origen levantino del cardenal Payá y Rico, que (junto con el final) constituye sin duda el pasaje más inequívocamente político del texto:

\footnotetext{
Si allá donde entre rosas y claveles arrastra el Turia sus revueltas ondas, nuestras manos talasen los jardines que plantaron los suyos, y aman ellos, su labio, al rostro, de desprecio llenas una tras otra injuria nos lanzaran -¡Bárbaros!- exclamando.
}

Castro: documentación biográfica y bibliografía crítica (1837-1990), A Coruña, Fundación Barrié de la Maza, 1991, p. 37-38). 


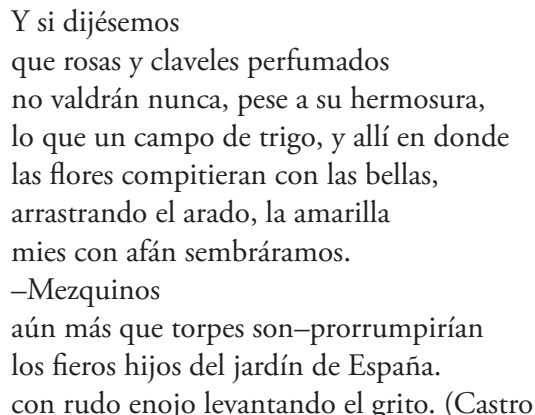

Y si dijésemos

que rosas y claveles perfumados

no valdrán nunca, pese a su hermosura,

lo que un campo de trigo, y allí en donde

las flores compitieran con las bellas,

arrastrando el arado, la amarilla

mies con afán sembráramos.

-Mezquinos

aún más que torpes son-prorrumpirían

con rudo enojo levantando el grito. (Castro, En las orillas... 485)

Además de culpar a quienes, con su silencio, son cómplices del abuso y de la injusticia, la voz injuria al cardenal foráneo, a quien el texto por fin identifica como principal agresor de la naturaleza y, por extensión, de Galicia. La relevancia de la estrofa viene dada por la especularidad (acentuada por su equivalente posición estructural) con respecto al pasaje ya comentado al comienzo de este artículo, en donde el bosque era descrito como si fuese un vergel. Muy sugerente es también la polaridad que el texto establece entre un nosotros y un ellos («los fieros hijos del jardín de España»). Característica del ideario nacionalista, la oposición apunta, en este caso, al contraste entre el paisaje atlántico, vinculado al imaginario céltico, y el referente levantino y sureño de la Península, blanco de una posible proyección orientalista. Como ha estudiado López García (419-420), el elemento diferencialista del orientalismo español reside precisamente en su proyección hacia el pasado árabe del territorio peninsular. En su consideración de que el paisaje del Norte es más agreste y menos civilizado, pero también más fresco y puro que el del mediterráneo, la autora pretende consagrar de paso la superioridad de la geografía norteña frente a la sureña, ideario de amplias resonancias en la historiografía gallega coetánea y especialmente en la obra de Manuel Murguía. En este juego relacional, la estrofa del poema puede leerse como una condensación del manifestario prólogo de Cantares gallegos (1863):

eu que visitei os celebrados arredores de Alicante, donde os olivos co seu verde escuro, sembrados en fila e de raro en raro, parecen chorar de verse tan solitarios, e vin aquela famosa horta de Murcia, tan nomeada e tan alabada, e que, cansada e monótona como o resto daquel paíse, amostra a súa vexetación tal como paisaxes pintados nun cartón con árbores postos simétricamente e en carreiriños para divertisión dos nenos, eu non podo menos de indignarnie cando os fillos desas provincias que Dios favoreceu en fartura, pero non na belleza dos campos, búlranse desta Galicia competidora en clima e galanura cos países máis encantadores da terra, esta Galicia donde todo é espontáneo e donde a man do home cede o seu posto á man de Dios. (Castro, Cantares... 489)

En ambos pasajes, la concepción providencialista de la existencia gallega se corresponde con un esencialismo étnico de fundamento paisajístico (López Sández). En justa correspondencia, la belleza natural llega a concebirse como el más logrado fruto de la mano de Dios. Pero en los veinte años que distan entre 
uno y otro texto la autora ha ido forjando una poética cada vez más refractaria al consenso y a los saberes comunes. Es como si, una vez reconocido su propio papel en el renacimiento cultural de Galicia, su obra testimoniase la distancia que media entre la vocación poética y los efectos comunitarios de esa vocación. Si consideramos, con el filósofo Jacques Rancière, que la política es la fuerza capaz de romper con el acuerdo y con los saberes consensuales, puede afirmarse que hay un sentido innegablemente político en "iJamás lo olvidaré...!», sin que resulte del todo claro que en él haya un sentido social.

En conformidad con la peculiar concepción rosaliana de la justicia, manifestada en poemas como "A xusticia pola man» (Castro, Follas... 306), el texto devuelve la agresión empuñando las armas del enemigo: es decir, dirigiendo el espíritu de Cruzada contra la Iglesia institucional española. Ningún pasaje de Rosalía de Castro permite esclarecer con nitidez el significado concreto que atribuía al Oriente Próximo en su obra, pero hay un mensaje en el poema que resuena con relativa claridad: allí donde la Iglesia oficial renuncia a su dimensión espiritual en favor de los intereses del mundo, la poesía es el único lugar donde el ser humano puede volver a vincularse de un modo pleno y auténtico con la sacralidad. Progresivamente separadas las esferas política y religiosa en el espacio social, lo sagrado vuelve a resonar, como por vez primera, en el discurso poético sobre la naturaleza. He ahí la paradoja de la constitución moderna -cabe decir, romántica- de la lírica: resulta ser, con respecto a lo sagrado y al mundo natural, simultáneamente un acto de despedida y de bienvenida (Leyte Coello).

Del alejamiento del texto con respecto a cualquier concepción ortodoxa de la fe habla ${ }^{7}$, entre otros factores, la caracterización druídica del bosque, donde la autora superpone el paganismo panteísta del imaginario celta con la presencia de símbolos como la resurrección, empleados en sentido etnorreligioso. Refiriéndose a la resurrección de Cristo, Nuala C. Johnson ha explicado el modo en el la escatología cristiana hace converger el tiempo individual con el tiempo universal:

in the Judaeo-Christian tradition is the importance of both individual and cosmic eschatology which combines the circularity of liturgical time practised through religious ritual (for instance the liturgical repetition of the birth, life, death and resurrection of the Lord in the Mass) with an acceptance of the linear time of history where the world is created only once and will experience one end, with one Last Judgment. (103)

Lo sugerente es que en el texto rosaliano Lázaro reemplace a Jesucristo como figura privilegiada para ejemplificar el renacer espiritual de la nación

7 . En la poesía peninsular del siglo XIX y principios del XX tal vez sea el religioso mallorquín Costa i Llobera el autor más ajustado a la ortodoxia en el empleo de motivos relacionados con el Oriente católico. El imaginario oriental atraviesa, de hecho, su obra, desde el temprano «El pi de Formentor», poema encabezado por el epígrafe Electus ut cedri (Miquel Costa i Llobera, Obres completes, Barcelona, Selecta, 1947, p. 17), hasta su libro de madurez Visions de Palestina (p. 167-191) o el poema dedicado "A Mossèn Llorenç Riber i Campins, mestre en Gai Saber» (p. 105). 
humillada. La elección tiene su origen en la parábola de Lucas (16, 19-31), que presenta al personaje como un pobre enfermo que sobrevive comiendo las migajas que caen de la mesa del rico. En virtud de la justicia divina, tras la muerte se invertirá la suerte de ambos y únicamente Lázaro encontrará un lugar en el cielo. La parábola habilita una equiparación metafórica entre el pobre y las comunidades de excluidos, razón por la cual, tomando como objeto a las mujeres, había sido usada en otros lugares del corpus rosaliano, como el prólogo de la novela La hija del mar. (Castro 48)

Pero acaso la mayor heterodoxia de «iJamás lo olvidaré...!» sea de carácter poético y descanse en su rechazo a la inflexión tonal de la épica o del himno. La voz nombra el significado de la pérdida para la comunidad, pero el registro se compadece mejor con otros géneros de fundamento emotivo y de enunciación primo-personal, especialmente con la oda y con la elegía. Una elección que se encuentra en perfecta consonancia con el sentido del poema, una auténtica enmienda a la totalidad del mundo, donde son condenados el agresor del bosque, quien consiente la agresión y quien ignora su verdadero significado ("Perdónales, / Señor, porque no saben lo que dicen» (Castro, En las orillas... 486). Es decir, donde únicamente es salvado el sujeto de la enunciación, revestido de las resonancias místicas del mártir. Y sin embargo, la vía negativa emprendida por la última poesía rosaliana limita el peligro de la solemnidad. Uno de los efectos de esta reticencia es el hecho de que el carácter monumental atribuido a la naturaleza no descanse tanto en los árboles como en las ruinas de los árboles. Todos ellos, no solo los castańos, vienen a ser «cedros de nuestro Líbano». Es decir, solo existen en la imaginación y en la memoria, materializadas en virtud de la $\mathrm{voz}^{8}$. Al igual que en "Los robles», el campo de batalla es un escenario espectral, cruzado por «el cuervo que grazna» y «el lobo que aúlla» (Castro, En las orillas... 477). En consecuencia, el eje temporal no es el ayer espléndido, sino el hoy desolado, simultáneamente su revelación y su contrario. Como sus admirados poetas místicos, la escritora parece situar el único fundamento de la pertenencia en la pérdida. Quien habla en el busca un eco, pero enuncia su voz desde el vacío: «¿Por qué gime / así importuna esa mujer?» (Castro, En las orillas... 486).

La potencia de «jamás lo olvidaré...!» emana, pues, de su capacidad para hacernos conscientes de una paradoja: símbolo de lo primordial para la comunidad, el árbol puede ser objeto de un corte capaz de cancelar su significado identitario. Pero esa interrupción violenta es la que crea la conciencia

8. La introducción del árbol en los jardines gallegos es casi contemporánea a la escritura del poema, como se deduce del catálogo de árboles singulares de Galicia que fecha en 130 ańos el Cedrus libani del Pazo de Lourizán y en 100 el conjunto de cedros del Himalaya del jardín de Vicenti, ambos situados en la provincia de Pontevedra (Antonio Rigueiro Rodríguez, José Gaspar Bernárdez Villegas y Carlos Rodríguez Dacal, Árbores e formacións senlleiras de Galicia, Santiago de Compostela, Xunta de Galicia, 2008, p. 6-8). Estos datos parecen confirmar el uso primordialmente literario y simbólico de este referente por parte de Rosalía de Castro. Agradezco al profesor Emilio Díaz Varela su ayuda en el acceso a fuentes valiosas para el conocimiento del patrimonio arbóreo de la Galicia del siglo XIX. 
del valor inmemorial del árbol. Es así como el poema adquiere la capacidad de revelación de un árbol centenario, al tiempo que funciona como un arma de guerra. Tan ardoroso como «On Jordan's Banks» de Byron, su uso desesperado de la escatología cristiana fuerza incluso la equiparación de la hablante con Cristo y la de Galicia con Lázaro. El poema resulta ser, en fin, un texto lleno de fuerza emotiva, y un artefacto capaz de condensar múltiples estratos del conocimiento sobre la relación entre la cultura y la naturaleza.

La autora, que en los últimos años de su vida decidió distanciarse de los círculos del Rexionalismo hegemónico (Moreiras Menor, Davies), parece escribir aquí desde una extrema libertad. El «mártir» a quien no le fue dado cumplir su obra de regeneración en El primer loco adquiere el mismo rostro que la «importuna mujer» de En las orillas del Sar. De algún modo, ambos vienen a confundirse con la anciana de Follas novas, la mujer desconocida por los suyos, que vivía sola en medio del bosque, en una casa de paredes de cedro. Como si se tratase de una heroína trágica, sus palabras ya no son escuchadas, y cuando son escuchadas, no son creídas.

En su análisis de las metáforas en los discursos científicos sobre la naturaleza, David Demeritt dibujó una oposición entre la historia ambiental-que sostiene la agencialidad de la naturaleza- y la geografía cultural -que apela al carácter socialmente construido del paisaje. Analizado a la luz de esta dialéctica, el poema parece dar la razón a los historiadores ambientales, para quienes la naturaleza "reminds us that there are different forces at work in the world and not all of them emanate from humans.» (Worster 292-293) Son esas fuerzas no humanas las que generan en la voz del poema una profunda conmoción, las que orientan la dicción hacia lo sagrado y las que acentúan el aislamiento del sujeto con respecto a quienes han quedado impunes a pesar de vulnerar el secreto del bosque.

Por más que en «jJamás lo olvidaré...!» los árboles adquieran cualidades políticas, históricas y culturales, la voz no afirma que su perspectiva sea capaz de crear el valor del paisaje, y el texto actúa como un indicio del esplendor perdido que el sujeto desea revelar. Fijándose en estas características de su producción, autoras como Davies y García Candeira han llevado a cabo interpretaciones de la obra de Rosalía de Castro a la luz de la ecocrítica, reconociendo el modo en que su obra concede al mundo natural una agencialidad relativamente infrecuente en su contexto histórico. La autora parece decirnos, en suma, que no es posible agotar el sentido de la naturaleza y que el último capítulo de la historia universal del bosque se oculta en las anillas concéntricas del cedro, un árbol del cual François Mauriac afirmó que extiende más allá de sus fronteras su misteriosa sombra ("bien au-delà de ses étroites frontières leur ombre mystérieuse», 7). 


\section{Obras citadas}

Abukhalil As'ad, Historical Dictionary of Lebanon, Lanham, The Scarecrow Press, 1998. Barreiro Fernández Xosé Ramón, Murguía, Vigo, Galaxia, 2012.

Byron George, The Works of Lord Byron. 4, Londres, John Murray, 1847.

Cabo Aseguinolaza Fernando, «Topografía y alegoresis en El primer loco, de Rosalía de Castro ", Revista de Estudios Hispánicos, no 50/1, 2016, p. 217-238.

Cabo Aseguinolaza Fernando y María do Cebreiro Rábade Villar, «"Tipos de Galicia. El cadista", de Antonio Neira de Mosquera», Boletín de la Biblioteca de Menéndez Pelayo, no LXXXIX, 2013, p. 199-216.

Castro Rosalía de, La hija del mar, en Marina Mayoral, Obras completas. 1, Madrid, Turner, 1993, p. 43-213.

Castro Rosalía de, Cantares gallegos, en Marina Mayoral, Obras completas. 1, Madrid, Turner, 1993, p. 483-641.

Castro Rosalía de, Flavio, en Marina Mayoral, Obras completas. 1, Madrid, Turner, 1993, p. 217-463.

Castro Rosalía de, Follas novas, en Marina Mayoral, Obras completas. 2, Madrid, Turner, 1993, p. 251-442.

Castro Rosalía de, En las orillas del Sar, en Marina Mayoral, Obras Completas. 2, Madrid, Turner, 1993, p. 443-553.

Coll y Vehí José, Compendio de Retórica y Poética ó nociones elementales de literatura, Barcelona, Imprenta del diario de Barcelona, 1875.

Costa i Llobera Miquel, Obres completes, Barcelona, Selecta, 1947.

Davies Catherine, Rosalia de Castro no seu tempo, Vigo, Galaxia, 1987.

Davies Catherine, «El mundo natural en Flavio» (Ponencia presentada en Un arma en el fango. I Seminario internacional sobre la narrativa de Rosalia de Castro. Santiago de Compostela, 22 de octubre de 2012).

Demeritt David. «The Nature of Metaphors in Cultural Geography and Environmental History», Progress in Human Geography, no 18.2, 1994, p. 163-185.

Fernández y Oliva Dalmiro, Elementos de Preceptiva literaria (Retórica y Poética), Palencia, Imprenta y Librería de Abundio Z. Meléndez, s. d.

Fernández Sánchez José María y Francisco Freire Barreiro, Santiago, Jerusalén, Roma, diario de una peregrinación a estos y otros santos lugares de España, Francia, Egipto, Palestina, Siria e Italia, en el año del jubileo universal de 1875, Santiago de Compostela, Imprenta del Boletín Eclesiástico, a cargo de D. Andrés Fraile, 1881-1884.

Holgado y Toledo Francisco, Literatura elemental (Retórica y Poética), Murcia, Tipografía de Antonio Molina, 1879.

Foucault Michel, Las palabras y las cosas. Una arqueología de las ciencias humanas (Trad. Elsa Cecilia Frost), México, Siglo XXI Editores, 2007.

García Candeira Margarita, «O ser natural e a súa dialéctica coa cultura en Rosalía de Castro", Actas do Congreso Rosalía de Castro no século XXI. Unha nova ollada, en Anxo Angueira, Rosario Álvarez, Dolores Vilavedra y María do Cebreiro Rábade Villar, Santiago de Compostela, Consello da Cultura Galega. 2014, p. 101-116. 
Johnson Nuala C., «From Time Immemorial: Narratives of Nationhood and the Making of National Space», en Jon May y Nigel Thrift, Timespace. Geographies of Temporality, Londres, Routledge, 2001.

León Luis de, Cantar de los Cantares, en Antolín Merino, Obras del M. Fr. Luis de León de la Orden de San Agustín, reconocidas y cotejadas con varios manuscritos auténticos. El Cantar de los Cantares con otras obras inéditas, Madrid, Imprenta de la Hija de Ibarra, 1806.

León Fray Luis de, Cantar de cantares de Salomón, en Javier San José Lera, Biblioteca Virtual Miguel de Cervantes [En línea]. Cervantes Virtual [Página consultada el 5 de febrero de 2014]. Accesible en: http://www.cervantesvirtual.com/obra-visor/ cantar-de-cantares-de-salomon--0/html/

Leyte Coello Arturo, «Introducción», en Juan Barja, Poesía completa de Rosalía de Castro, Madrid, Abada, 2009.

López García Bernabé, Orientalismo e ideología colonial. Arabismo español (1840-1917), Granada, Universidad de Granada, 2012.

López Sández María, Paisaxe e nación: a construción discursiva do territorio, Vigo, Galaxia, 2007.

López Aurora y Andrés Pociña, Rosalia de Castro: documentación biográfica y bibliografía crítica (1837-1990), A Coruńa, Fundación Barrié de la Maza, 1991.

Malkki Liisa, «National Geographic: The Rooting of Peoples and the Territorialization», Becoming National. A Reader, New York \& Oxford, Oxford University Press, 1996, p. 434-53.

Martín Asuero Pablo, España y el Líbano, 1788-1910. Viajeros, diplomáticos, peregrinos, e intelectuales (con un apéndice sobre el Libano actual), Madrid, Miraguano Ediciones.

Mauriac François, «Préface», en Jacques Nantet, Histoire du Liban, Paris, Les Éditions de Minuit, 1963, p. 7-10.

Mialdea Baena José Antonio, La recepción de la obra literaria de San Juan de la Cruz en España durante los siglos XVII, XVIII y XIX (Tesis doctoral), Córdoba, Universidad de Córdoba, 2002.

Mikesell Marvin W., "The Deforestation of Mount Lebanon», The Geographical Review, no 40.1, 1969, p. 1-28.

Moreiras Menor Cristina, «El secreto revelado y los horizontes del nacionalismo gallego en Rosalía de Castro", Revista Hispánica Moderna no 52.2, 1999, p. 322-340.

Mudarra y Párraga Prudencio, Lecciones de Literatura general y Literatura española, Sevilla, Gironés, 1876.

Murguía Manuel, Los precursores, A Coruña, La Voz de Galicia, 1885.

Neira de Mosquera Antonio, «Espańa árabe», El Recreo Compostelano, no 2, 26 de enero de 1842, p. 17-20.

Neira de Mosquera Antonio, «Jerusalén. El Santo Sepulcro», El Recreo Compostelano, no 6, 26 de marzo de 1842, p. 81-85.

Nora Pierre, «Between Memory and History: Les Lieux de Mémoire», Representations, no 26, 1989, p. 7-25.

Özkirimli Umut, Contemporary Debates on Nationalism. A Critical Engagement, Houndmills, Palgrave McMillan, 2005. 
Pavesi Pablo E, «Foucault y la vida. Historia de la biología y genealogía de la biopolítica», Cuadernos de pensamiento biopolítico latinoamericano, no 1, 2013, p. 93-96.

Pérez Reoyo Narciso, Viaje a Egipto, Palestina y otros paises del Oriente, Lugo, Imprenta de Soto, a cargo de Juan María Bravos, 1875.

Rancière Jacques, El desacuerdo. Política y filosofía, Buenos Aires, Nueva Visión, 1996. Rigueiro Rodríguez Antonio, José Gaspar Bernárdez Villegas y Carlos Rodríguez Dacal, Árbores e formacións senlleiras de Galicia, Santiago de Compostela, Xunta de Galicia, 2008.

Rivadeneyra Adolfo, Viaje de Ceylan a Damasco, Golfo Pérsico, Mesopotamia, Ruinas de Babilonia, Ninive y Palmira y Cartas sobre la Siria y la Isla de Ceylán, Madrid, Imprenta y Estereotipia de M. Rivadeneyra, 1871.

Rodríguez Francisco, Estranxeira na súa patria. A obra de Rosalia de Castro de onte a hoxe, A Coruña, AS-PG, 2011.

Salem Elise, Constructing Lebanon. A Century of Literary Narratives, Gainesville, University Press of Florida, 2003.

Tennyson Alfred, Maud, and Other Poems, London, Edward Moxon \& Co., 1859.

Smith Anthony, The Ethnic Origins of Nations, Oxford, Blackwell, 1986.

Worster D., "Appendix: Doing Environmental History», en D. Worster, The Ends of the Earth-Perspectives on Modern Environmental History, New York, Cambridge University Press, 1988, p. 289-307.

Zafer Chaaban Nadia, La imagen del Líbano en los escritos de viaje españoles de la segunda mitad del siglo XIX (Tesis doctoral), Madrid, Universidad Autónoma, 1988. 